\title{
Zusammenfassung.
}

I. Es gibt kernlose Zellen, die eine meßbare Sauerstoffatmung haben, nämlich die roten Blutzellen der Säugetiere.

Der Nachweis gelingt nicht mit Sicherheit an den roten Blutzellen normaler erwachsener Menschen; dagegen wurde in den Erythrocyten der Kaninchen ein Material gefunden, mit dem einwandfreie Resultate zu erhalten sind.

Die Größe der Sauerstoffatmung war sehr verschieden; es stellte sich heraus, daß sie sich vorhersagen läßt auf Grund des histologischen Bildes: sie geht parallel der Basophilie, d. h. in den untersuchten Fällen der Jugend der Zellen. Hierbei bleibt unentschieden, ob nur die basophilen oder alle Erythrocyten atmen. Eine große Zahl basophiler Zellen ohne kernhaltige Erythrocyten findet man in der Regel bei jungen (5-20 Tage alten) Kaninchen.

II. Die (kernhaltigen) Erythrocyten normaler ausgewachsener Vögel haben eine sehr erhebliche Sauerstoffatmung; jedenfalls ist sie von einer ganz andern Größenordnung als die der Erythrocyten normaler erwachsener Säugetiere.

\section{Nachtrag zu «Die Schwefelbestimmung im Urin». \\ Von}

Emil Abderhalden und Casimir Funk.

(Der Redaktion zugegangen am 19. Februar 1909.)

Wir haben in Anlehnung an das Verfahren von Pringsheim eine Methode zur Bestimmung von Schwefel im Urin mitgeteilt, die sehr zuverlässige Resultate gibt und wenig Zeit in Anspruch nimmt. Nachträglich finden wir, daß G. Modrak ow ski ${ }^{1}$ ) ebenfalls Natriumsuperoxyd angewandt und die Ausführung der Schwefelbestimmung im Urin abgekürzt hat. Die von uns angegebene Methode dürfte jedoch vorzuziehen sein. Der Vollständigkeit wegen sei auch noch angeführt, daß die Schwefelbestimmung im Urin nach Schulz von Artur Konschegg ${ }^{2}$ ) modifiziert worden ist.

1) G. Modrakowski, Über die Schwefelbestimmung im Harn mittels Natriumsuperoxyd. Diese Zeitschrift, Bd. XXXVIII, S. 562, 1903.

$\left.{ }^{2}\right)$ Artur Konschegg, Zur Bestimmung des Gesamtschwefels im Harne. Pflügers Archiv, Bd. CXXIII, S. 274, 1908. 